\title{
Sepsis tuberculosa gravissima. Una presentación infrecuente en paciente con tratamiento inmunosupresor
}

'Servicio de MedicinaUnidad de Enfermedades

Respiratorias Hospital Regional de Talca.

${ }^{2}$ Unidad de Cuidados Intensivos Hospital Regional de Talca. ${ }^{3}$ Servicio de Radiología Hospital Regional de Talca. ${ }^{4}$ Escuela de Medicina Universidad de Talca.

Recibido el 14 de enero de 2011, aceptado el 9 de mayo de 2011

Correspondencia:

Dr. Rafael Silva O.

Calle 2 Norte 360 Talca Fono $71-412716$ Fax 71- 209306

E-mail: rafaelsilvao@gmail. com

\author{
RAFAEL SILVA $^{1,4}$, JUAN JARA ${ }^{2}$, TULIO SOTO ${ }^{1}$, PEDRO SEPÚLVEDA ${ }^{3}$
}

\section{Severe disseminated tuberculosis in a patient on immunosuppressive treatment. Report of one case}

Patients on immunosuppressive therapy are at increased risk of developing tuberculosis. We report a 39-year-old female with an ulcerative colitis receiving prednisone, azathioprine and azulfidine that was admitted to hospital due to fever, anemia, diarrhea and hematochezia. A chest CT scan showed multiple miliary micronodular images diffusely distributed and mediastinal enlarged lymph nodes with central necrosis, suggestive of disseminated tuberculosis. Antituberculous treatment was started but discontinued and the patient was treated as a bronchiolitis obliterans with methylprednisolone pulses and discharged. She was readmitted in shock one week later and died. After her death cultures for Mycobacterium tuberculosis were informed as positive. The clinical picture of the patient is known as sepsis tuberculosa gravissima.

(Rev Med Chile 2011; 139: 774-778).

Key words: Methylprednisolone; Shock, septic; Tuberculosis, miliary.

\section{I}

a tuberculosis (TBC) es una enfermedad infecto-contagiosa causada por el Mycobacterium tuberculosis, con una incidencia en Chile de $18,4 \%$ o el año 2003 y de $13,6 \%$ el año 2008, encaminándose a la fase epidemiológica denominada "de eliminación"1.

Está demostrado que los pacientes con terapia inmunosupresora tienen mayor riesgo de contraer tuberculosis, respecto la población general. En estos pacientes es esencial realizar el diagnóstico precoz, sin embargo los bacilos ácido-alcohol resistentes se detectan en el esputo en un número limitado de pacientes, por lo tanto el desafío del diagnóstico precoz puede ser abordado con la ayuda de la imagenología ${ }^{2}$. Las características imagenológicas de la TBC son muy variables, pero en una proporción importante de casos estas imágenes pueden ser muy orientadoras respecto a la presencia de esta enfermedad, particularmente en aquellas obtenidas por tomografía computada de tórax multicorte (TCM).
Un hallazgo significativo en la TCM son las imágenes denominadas "árbol en brote", que consisten en múltiples opacidades lineales que puede representar, entre otras causas, la diseminación broncógena de la infección tuberculosa. Estas imágenes representan los focos de necrosis caseosa que afectan a los bronquiolos terminales y respiratorios, pero no son patognomónicas de TBC pulmonar y pueden ser encontradas en patologías como bronquiolitis post-viral, por inhalación de gases tóxicos y por polvos inorgánicos, entre otras ${ }^{3}$.

Presentamos el caso de una paciente en tratamiento inmunosupresor por colitis ulcerosa que evoluciona con una infección tuberculosa diseminada de evolución rápidamente fulminante, sin presentar otro foco infeccioso concomitante.

\section{Caso clínico}

Mujer de 39 años, portadora de colitis ulcerosa diagnosticada por cuadro clínico y biopsia de 
colon en diciembre de 2008, iniciando en enero de 2009 tratamiento con sulfasalazina 3 gramos/ día, prednisona $20 \mathrm{mg} /$ día y azatioprina $100 \mathrm{mg} /$ día. Continuó sintomática pese al tratamiento y control regular, necesitando dos hospitalizaciones el año 2009 por reactivación de la colitis ulcerosa. En junio del 2010 fue nuevamente ingresada por cuadro febril de 15 días de evolución asociado a hemorragia digestiva baja, anemia severa y diarrea. Una nueva colonoscopía demostró una pancolitis, descartándose un megacolon tóxico u otra complicación abdominal. La radiografía de tórax mostró múltiples nódulos finos, de aproximadamente $3 \mathrm{~mm}$, de distribución azarosa, bilateral, mayor a derecha, con patrón miliar (Figura 1) y una TCM pulmonar que mostró imágenes micronodulares de aspecto miliar, mal definidas, de distribución difusa y opacidades periféricas con morfología de "árbol en brote" (Figura 2) más adenopatías mediastínicas con necrosis central (Figura 3). Las imágenes descritas eran muy sugerentes de TBC pulmonar activa con diseminación hematógena y bronquiolar. La paciente no presentaba tos, expectoración ni hemoptisis pero se constató una insuficiencia respiratoria aguda parcial.

Basados en los hallazgos imagenológicos se comienzó tratamiento antituberculoso el 13 de julio de 2010, pese a tener baciloscopias de esputo negativas. Ese mismo día se acentuó la falla respiratoria aguda como consecuencia de un neumotórax secundario a la instalación de un catéter venoso

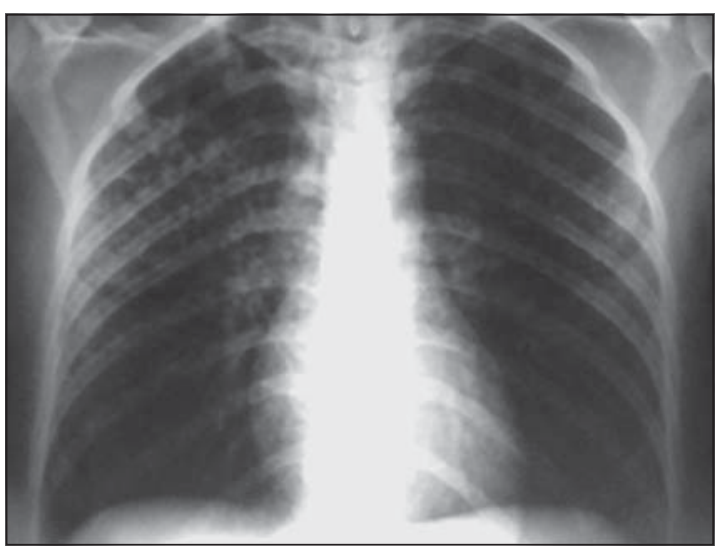

Figura 1. Radiografía de tórax AP que muestra presencia de múltiples nódulos finos, de aproximadamente $3 \mathrm{~mm}$. de diámetro, de distribución heterogénea y bilateral mayor a derecha, con aspecto de patrón miliar. central. Se instaló un tubo de drenaje pleural y ante la eventual necesidad de ventilación mecánica fue trasladada a una clínica privada de Santiago, al no disponer de camas de UCI en Talca. En Santiago, el equipo médico interpretó el caso como bronquiolitis obliterante con neumonía organizada (BOOP) y suspendió el tratamiento antituberculoso. Inició pulsos de metilprednisolona 1 gramo/día por 3 días, mantuvo prednisona $1 \mathrm{mg} / \mathrm{kg}$ asociado a azatioprina $100 \mathrm{mg} /$ día, suspendió sulfasalazina y retiró drenaje pleural. La evolución fue favorable, sin requerir ventilación mecánica, dándose de alta el 27 julio de 2010.

El 5 de agosto consultó en servicio de urgencia del hospital regional de Talca por fiebre $39^{\circ} \mathrm{C}$ de cuatro días de evolución, taquipnea, taquicardia e hipotensión, manejándose inicialmente en el servicio de medicina y posteriormente en la unidad de cuidados intermedios. Por la persistencia del compromiso hemodinámico y la sospecha de un shock séptico, ingresó a la unidad de cuidados

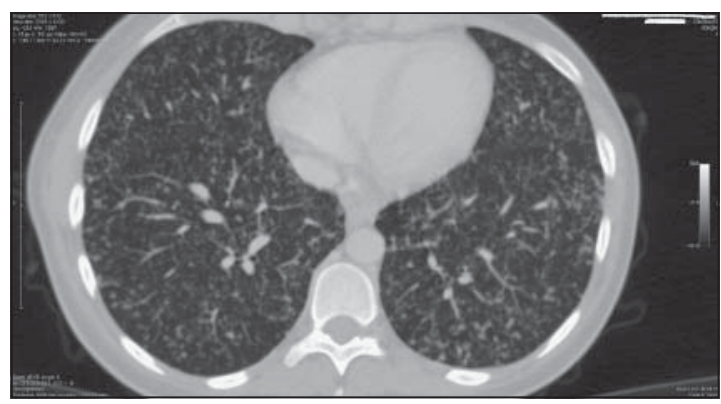

Figura 2. Tomografía computada de tórax de alta resolución, en ventana pulmonar, que muestra imágenes micronodulares de patrón miliar mal definidas de distribución difusa y opacidades periféricas con morfología de "árbol en brote".

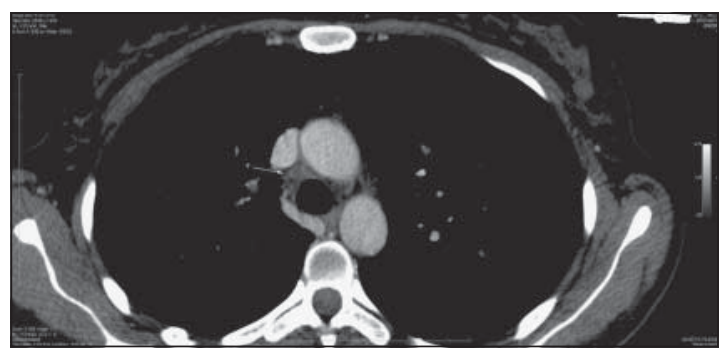

Figura 3. Tomografía computada de tórax, en ventana mediastínica, que muestra imagen (flecha) de adenopatía con centro necrosado. 
Tabla 1. Exámenes de laboratorio: pruebas hepáticas, proteína C reactiva, leucocitos y baciliformes, ácido láctico, albúmina y protrombina, entre el 10 y el 19 de agosto 2010

\begin{tabular}{|lccccc|}
\hline & $\mathbf{1 0}$ Agosto & $\mathbf{1 3}$ Agosto & $\mathbf{1 6}$ Agosto & $\mathbf{1 8}$ Agosto & $\mathbf{1 9}$ Agosto \\
\hline Bilirrubina Total (mg./dL) & 2,39 & 3,43 & 3,9 & 6,07 & 8,05 \\
\hline Bilirrubina Directa (mg./dL) & 2,23 & 3,10 & 3,25 & 5,77 & 7,9 \\
\hline SGOT (U/L) & 77 & 21 & 15 & 9 & 15 \\
\hline SGPT (U/L) & 44 & 4 & 7 & 2 & 1 \\
\hline Proteína C Reactiva (mg./L) & 428 & 196 & 109 & 309 & 410 \\
Creatinina (mg./dL) & 2,09 & 3.12 & 3,9 & 2,9 & 3.3 \\
Leucocitos (mm.3) & 2.080 & 2.720 & 2.220 & 1.150 & 1.100 \\
\hline Baciliformes (\%) & 25 & & & & 25 \\
Acido Láctico (mg./dL) & 32 & 16.5 & 11 & 17 & 17 \\
Albúmina (gr./dL) & 1,9 & & & 2,6 & 70 \\
\hline Protrombina (\%) & 52 & 76 & 74 & 65 & 70 \\
\hline
\end{tabular}

intensivos a los tres días de admisión. El 10 de agosto fue evaluada por el equipo de respiratorio del hospital regional de Talca y se decidió reiniciar el tratamiento antituberculoso. Evolucionó con falla respiratoria progresiva, requiriendo ventilación mecánica invasiva, apoyo hemodinámico con cristaloides y coloides para mantener presión arterial media estable y presión venosa central hasta $12 \mathrm{~mm} / \mathrm{Hg}$ más noradrenalina hasta 0,55 gamma/ $\mathrm{kg} / \mathrm{min}$ y adrenalina hasta $0,40 \mathrm{gamma} / \mathrm{kg} / \mathrm{min}$.

Los parámetros de laboratorio (Tabla 1) y hemodinámicos eran compatibles con un shock séptico, evolucionando refractaria a las medidas señaladas, presentando falla orgánica múltiple, ante lo cual fallece el 19 agosto 2010. Los cultivos bacterianos de secreciones bronquiales, orina $y$ sanguíneos fueron todos negativos. No se demostró otro foco infeccioso bacteriano ni una complicación abdominal derivada de la colitis ulcerosa.

Las baciloscopías de aspirado traqueal (4) fueron negativas, pero el cultivo de Koch de los 30 días fue positivo para 20 colonias de Mycobacterium tuberculosis, resultado recibido post-mortem.

\section{Discusión}

La tuberculosis pulmonar se puede presentar de forma aguda (duración síntomas $\leq 30$ días), con características de TBC miliar o de disemi- nación bronquiolar, pudiendo ambas progresar en severidad y requerir manejo en unidades de cuidados intensivos entre el 1 y $3 \%$ de los casos, siendo infrecuente la presentación como sepsis o shock ${ }^{4}$.También la diseminación hematógena del Mycobacterium tuberculosis puede ocurrir con o sin enfermedad miliar, conocida esta última como septicemia Landouzy o "sepsis tuberculosa acutissima"s.

Cuando la tuberculosis se presenta en forma diseminada puede cursar como una "sepsis tuberculosa gravissima", cuadro caracterizado por presentar shock séptico y falla orgánica múltiple ${ }^{5}$. La sepsis asociada a TBC es más común en pacientes inmunodeprimidos, que pueden rápidamente progresar a falla multisistémica antes que cualquier signo de TBC sea evidente ${ }^{6}$, pero algunas formas agudas, especialmente la forma "gravissima" puede provocar la muerte en pacientes inmunocompetentes ${ }^{7}$.

La variante séptica de la tuberculosis es poco frecuente en pacientes inmunocompetentes y se asocia frecuentemente a tuberculosis miliar, pero se han descrito casos de sepsis tuberculosa severa sin TBC miliar ${ }^{8}$, donde el shock séptico asociado a la infección micobacteriana estaría relacionado con la producción de factor de necrosis tumoral $\mathrm{alfa}^{5}$, factor que juega un rol mayor en estos pacientes, el cual es estimulado por un componente de la pared celular del Mycobacterium tuberculosis denominado lipoarabinomannan, un glicolípido que 
puede enlazar leucocitos y modular la respuesta inmune ${ }^{9,10}$.También el Mycobacterium tuberculosis produce lipopolisacáridos que pueden provocar sepsis a través de un mecanismo semejante a las bacterias Gram (-), aunque el mecanismo exacto aún no está determinado ${ }^{11}$.

Por otro lado, en más de un tercio de los pacientes con TBC pulmonar, la TCM muestra consolidación lobar o multifocal y en la mitad de los pacientes se observa cavitación o derrame pleural mientras que el patrón miliar y las adenopatías mediastínicas se ven en menos de $10 \%$ de estos pacientes ${ }^{12}$. En algunos pacientes con insuficiencia respiratoria aguda es frecuente encontrar lesiones nodulares centrilobulillares pequeñas difusas tipo "árbol en brote", lo que es concordante con una diseminación broncógena de la $\mathrm{TBC}^{13}$. La presencia de estas opacidades se asocia con alta probabilidad a la pesquisa de Mycobacterium tuberculosis en el esputo $^{14,15}$.

Se ha documentado que los nódulos peribronquiolares intralobulares, observados en la anatomía patológica de pacientes portadores de TBC pulmonar, son los que se evidencian como opacidades tipo "árbol en brote" en la TCM, y esto ocurre porque la infección tuberculosa se localiza alrededor de los bronquiolos provocando una bronquiolitis infecciosa por el Mycobacterium tuberculosis ${ }^{16}$.

También las enfermedades inflamatorias intestinales tales como la enfermedad de Crohn, la colitis ulcerosa y el tratamiento con sulfasalazina, pueden provocar daño pulmonar ${ }^{17}$. En la colitis ulcerosa habitualmente se compromete la vía aérea central y es muy raro observar compromiso de la vía aérea pequeña ${ }^{18}$. Es frecuente que el daño pulmonar en la colitis ulcerosa se manifieste por bronquiolitis obliterante ${ }^{19}$ o por bronquiolitis obliterante con neumonía organizada $(\mathrm{BOOP})^{20}$. Sin embargo, estas lesiones no corresponden a las imágenes radiológicas observadas en esta paciente. También se han descrito casos aislados de BOOP con el uso de sulfasalazina, medicamento que usaba esta paciente, pero este cuadro se acompaña frecuentemente de eosinofilia en sangre periféri$\mathrm{ca}^{21}$, lo que tampoco se observa en este caso.

Hay pocos casos de sepsis tuberculosa gravissima descritos en la literatura. En una serie de 3 casos publicada el año $2002^{7}$ todos los pacientes fallecieron por falla multiorgánica, evolución que también presentó nuestra paciente.
En resumen, se comunica un caso de tuberculosis de diseminación broncogénica, en una paciente portadora de colitis ulcerosa en tratamiento inmunosupresor, que presenta una evolución séptica y falla orgánica múltiple asociada a su enfermedad tuberculosa, sin otros focos que expliquen su evolución, configurando el cuadro de sepsis tuberculosa gravissima, lo que finalmente le ocasiona la muerte.

En conclusión, nos parece razonable recomendar la búsqueda activa de la infección por $\mathrm{Myco-}$ bacterium tuberculosis en pacientes gravemente enfermos, especialmente en los inmunodeprimidos, con falla respiratoria aguda y shock séptico, sin foco aparente y con opacidades pulmonares difusas de distribución miliar o bronquiolar en la TCM, junto con también recomendar el inicio precoz de la terapia antituberculosa, lo que puede cambiar el pronóstico de una enfermedad tratable y curable.

\section{Referencias}

1. http://www.redsalud.gov.cl/portal/url/item/803048171a cc60f8e04001011f0148e2.pdf (acceso 30 de diciembre de 2010).

2. Lee KS, Im JG. CT in adults with tuberculosis of the chest: characteristic findings and role in management. AJR 1995; 164: 1361-7.

3. Andreu J, Cáceres J, Pallisa E, Martínez-Rodríguez M. Radiological manifestations of pulmonary tuberculosis. Eur J Radiol 2004; 51: 139-49.

4. Frame R, Johnson M, Eichenhorn M. Active tuberculosis in the medical intensive care unit: a 15-year retrospective analysis. Crit Care Med 1987; 15: 1012-4.

5. Jacob JT, Mehta AK, Leonard MK. Acute forms of tuberculosis in adults. Am J Med 2009; 122: 12-7.

6. Ahuja S, Phelps K. Hemodynamic confirmation of septic shock in disseminated tuberculosis. Crit Care Med 1992; 20: 901-3.

7. Michel P, Barbier C, Loubiere Y. Three cases of septic shock due to tuberculosis without HIV pathology. Intensive Care Med 2002; 28: 1827-8.

8. Bridged D, Bedimo R. Severe tuberculosis sepsis in an immunocompetent patient. Am J Med 2006; 119: e11e14.

9. Vadillo M, Corbella X, Carratala J. AIDS presenting as septic shock caused by Mycobacterium tuberculosis. Scand J Infect Dis 1994; 26: 105-6.

10. Strohmeier G, Fenton M. Roles of lipoarabinomannan in 
the pathogenesis of tuberculosis. Microbes and Infection 1999; 1: 709-17.

11. Matthay MA. Severe sepsis: a new treatment with both anticoagulant and antiinflammatory properties. N Engl J Med 2001;344:759-62

12. Chao-Jung W, Phd, Chui-Mei T, Jen-Dar C, Yi-Hong C, Cheng-Yen C, Chun Y. Computed Tomography Features of Acute Pulmonary Tuberculosis.. Am J Emerg Med 2004; 22: 171-4.

13. Dongil C, Kyung Soo L, Gee Young S, Tae Sung K, Jung $\mathrm{K}$, Chong $\mathrm{H}$, et al. Pulmonary tuberculosis presenting as acute respiratory failure: Radiologic findings. J Comput Assist Tomogr 1999; 23: 107-13.

14. Poey C, Verhaegen F, Giron J. High resolution chest CT in tuberculosis: evolutive patterns and signs of activity. J Comput Assist Tomogr 1997; 21: 601-7.

15. Kosada N, Sakai T, Uematsu H, Kimura H, Hase M, Noguchi $\mathrm{M}$, et al. Specific high-resolution computed tomo- graphy findings associated with sputum smear-positive pulmonary tuberculosis. J Comput Assist Tomogr 2005; 29: 801-4.

16. Murata K, Itoh H, Todo G. Centrilobular lesions of the lung: demonstration by high-resolution $\mathrm{CT}$ and pathologic correlation. Radiology 1986; 161: 641-5.

17. http://www.pneumotox.com/indexf.php?fich=drugs\&lg $=$ sp\&nf $=($ acceso 10 de marzo de 2011).

18. Camus P, Colby T. Respiratory manifestations in ulcerative colitis. Eur Respir Mon 2006; 34: 168-183.

19. Wilcox P, Miller R, Miller G. Airway involvement in ulcerative colitis. Chest 1987; 92: 18-22.

20. Swinburn C, Jackson G, Cobden I, Ashcroft T, Moritt G, Corris P. Bronchiolitis obliterans organising pneumonia in a patient with ulcerative colitis. Thorax 1988; 43: 7356.

21. Epler G. Drug-induced bronchiolitis obliterans organizing pneumonia. Clin Chest Med 2004; 25: 89-94. 